$63^{\text {ème }}$ Congrès de la SFCO, 03016 (2015)

DOI:10.1051/sfco/20156303016

(C) Owned by the authors, published by EDP Sciences, 2015

\title{
Atteinte plurifocale de syphilis secondaire : présentation d'un cas
}

\author{
Campana $\mathrm{F}^{*}$, Carvelli $\mathrm{J}^{* *}$, Fricain $\mathrm{J}-\mathrm{C}^{* * *}$, Vergier $\mathrm{B} * * * *$, Boralevi $\mathrm{F}^{* * * * *}$, Mazodier $\mathrm{K}^{* *}$, \\ Kaplanski G** \\ * Centre Massiliende la Face, 24 avenue du Prado, Entrée 1 entresol, 13006 Marseille \\ ** Service de Médecine interne, Hôpital de la Conception, 147 boulevard Baille, Assistance publique, Hôpitaux \\ de Marseille, 13005 Marseille \\ *** Pôle d'Odontologie et de santé buccale, Hôpital Pellegrin, Place Amélie Raba Léon, CHU de Bordeaux, \\ 33076 Bordeaux \\ **** Service de Pathologie, Hôpital Haut Lévêque, 1 avenue Magellan, CHU de Bordeaux, 33604 Pessac \\ ***** Service de Dermatologie Pédiatrique, Hôpital Pellegrin, Place Amélie Raba Léon, CHU de Bordeaux, \\ 33076 Bordeaux
}

La syphilis est une infection sexuellement transmissible (IST) liée au treponema pallidum (Janvier 2014). Elle touche 12 millions de personnes dans le monde/an (OMS 2009). Son diagnostic reste un challenge car les signes cliniques ne sont pas spécifiques, valant à la syphilis le surnom de "grande simulatrice ».

Nous rapportons ici le cas d'une atteinte plurifocale chez une patiente sans facteur de risque d'IST.

Une femme de 70 ans aux antécédents de zona intercostal et de cruralgie, sans traitement médicamenteux consultait pour des douleurs buccales d'apparition récentes. L'histoire de la maladie avait débuté 3 mois avant, avec une lésion génitale prurigineuse entre la grande et la petite lèvre droite étiquetée herpès et traitée par zelitrex® ${ }^{\circledR}$. L’examen endobuccal retrouvait des lésions ulcérées du bord droit de langue et érosives du palais osseux. Il existait des adénopathies cervicales. L'examen cutané objectivait un exanthème généralisé vésiculo-bulleux d'apparition rapide. Des biopsies étaient réalisées. Elles mettaient en évidence au niveau buccal une hyperkératose et un infiltrat lymphocytaire d'aspect lichénien, sans noter d'effet cytopathogène viral. Au niveau cutané on retrouvait un infiltrat péri vasculaire discret et une hyperkératose. Les immunofluorescences directes étaient négatives. L'hémogramme était sans anomalie. Il apparaissait une uvéite non granulomateuse de l'œil droit antérieure, qui devenait postérieure. La patiente était hospitalisée dans le service de médecine interne. Le bilan retrouvait le TPIE positif à 40,79, le VDRL positif à 64 et le VIH négatif. La ponction lombaire montrait une méningite lymphocytaire. La TDM cérébral et TAP étaient normales. Le diagnostic de syphilis secondaire avec atteinte neurologique était posé. L'interrogatoire renouvelé permettait de mettre en évidence la fréquentation de travailleur du sexe par le concubin avec la notion de chancre pénien mis sous silence. Une immunohistochimie était réalisée et retrouvait au niveau muqueux un important marquage de treponema pallidum. Un traitement par benzathine penicilline G IV (20 millions d'unités/j) était instauré pendant 14 jours. La guérison était obtenue l'ensemble des lésions. La patiente regagnait le domicile. Un suivi était instauré.

This is an Open Access article distributed under the terms of the Creative Commons Attribution License 4.0, which permits unrestricted use, distribution, and reproduction in any medium, provided the original work is properly cited. 
La syphilis est une IST liée à un spirochète, le treponema pallidum. La transmission peut être maternofoetale. Le nombre de cas a été multiplié par 10 en France entre 2000 et 2010 et est estimé à 500 cas/an en 2010 (Bouyssou 2011). La syphilis après une période d'incubation de 10-90 jours (Janvier 2014) évolue par plusieurs phases (primaires, secondaires, tertiaires) séparées par des phases de latence. Les lésions sont très polymorphes et évoluent selon le stade de la maladie rendant le diagnostic difficile, d'autant que l'expérience des praticiens est devenue faible. Les complications, notamment neuro méningées et ophtalmologiques, peuvent être graves (HAS 2007). La sérologie occupe une place centrale dans le diagnostic (Fahri 2008). On utilise un test spécifique comme le TPHA et un test non spécifique comme le VDRL (HAS 2007). Le traitement de première intention reste la benzathine penicilline G par voie IV (Janier 2014). En cas d'allergie, on utilise la doxycycline.

Nom et adresse du conférencier

Fabrice CAMPANA

Centre Massilien de la Face

24 avenue du Prado, Entrée 1 entresol

13006 Marseille (France)

f.campana@centremassiliendelaface.com 